\title{
Comparative efficacy of 5 surgical methods in the treatment of mitral regurgitation: a systematic review and network meta-analysis
}

\author{
Weimin Huang ${ }^{1}$, Biao Hou ${ }^{2}$, Yuhai Zhang ${ }^{2}$, Qin $\mathrm{Li}^{2}$, and Liang Wang ${ }^{2}$ \\ ${ }^{1}$ Baotou Clinical Medical College of Inner Mongolia Medical University \\ ${ }^{2}$ Affiliation not available
}

September 25, 2021

\begin{abstract}
Objective This study has been compared the effectiveness of different surgical methods in the treatment of mitral regurgitation (MR) in adults by using network meta-analysis method, so as to provide reference for clinical selection of the best surgical scheme. Methods The PubMed, EMBASE, the Cochrane Library, CNKI and Chongqing VIP Information databases were comprehensively searched until December 2020. We collected retrospective comparative studies on surgical procedures including 3D endoscopic mitral valve surgery(3D-MVS), robot assisted mitral valve surgery(R-MVS); totally thoracoscopic mitral valve surgery(T-MVS), small incision mitral valve surgery (M-MVS) and traditional thoracotomy mitral valve surgery(C-MVS). Addis1.16.8 software was used for network meta-analysis. Results A total of 31 studies were included, 12998 patients, involving 5 surgical methods. Network Meta analysis showed that: in terms of complications (OR: 0.65, 95\% CI: 0.13 to 3.00 , probability rank $=0.37$ ) and mitral regurgitation (OR:0.03, 95\%CI: 0.0 to 8315, probability rank=0.64), the 3D-MVS group had the lowest event rate. In terms of blood transfusion rate (OR: $0.55,95 \%$ CI: 0.16 to 1.84 , probability rank=0.45), T-MVS had the lowest event rate. In addition, with the exception of operation time and chest drainage, the R-MVS group has the best curative effect. Conclusion These minimally invasive surgery has their own advantages and disadvantages. Overall, 3D-MVS is most satisfactory, but more samples are needed.
\end{abstract}

Comparative efficacy of 5 surgical methods in the treatment of mitral regurgitation: a systematic review and network meta-analysis

Weimin Huang ${ }^{1}$, Biao Hou ${ }^{1}$, Qin $\mathrm{Li}^{1}$, Yuhai Zhang ${ }^{1}$, Liang Wang ${ }^{2}$

1. Baotou Clinical Medical College of Inner Mongolia Medical University;

2. Department of Cardiology, Baotou Central Hospital, Baotou 014040, China.

Correspondence to:

Liang Wang;huang_manlin2021@163.comPhone number: +86138-9086-2882

523, Angel Building, Baotou Central Hospital, Inner Mongolia Autonomous Region

【Key words \Minimally invasive surgery; Mitral valve; Thoracoscopy; Robot surgery; Traditional thoracotomy; Network meta-analysis

$¿$ This is the first network meta-analysis focused on different surgical treatments for mitral regurgitation to review all available literature.

¿. We employed rigorous methodology according to the Preferred Reporting Items for Systematic Reviews and Meta-Analyses statement, and used the Recommendations Assessment, using the software ADDIS 1.16.8 in the review process. 
¿Due to special circumstances, the thoracoscopy assisted or robot assisted surgery are not listed as separate surgical method to collect data for subgroup analysis.

\section{ABSTRACT}

Objective This study has been compared the effectiveness of different surgical methods in the treatment of mitral regurgitation (MR) in adults by using network meta-analysis method, so as to provide reference for clinical selection of the best surgical scheme. Methods The PubMed, EMBASE, the Cochrane Library, CNKI and Chongqing VIP Information databases were comprehensively searched until December 2020. We collected retrospective comparative studies on surgical procedures including $3 \mathrm{D}$ endoscopic mitral valve surgery(3D-MVS), robot assisted mitral valve surgery(R-MVS); totally thoracoscopic mitral valve surgery(TMVS), small incision mitral valve surgery (M-MVS) and traditional thoracotomy mitral valve surgery(CMVS). Addis1.16.8 software was used for network meta-analysis. Results A total of 31 studies were included, 12998 patients, involving 5 surgical methods. Network Meta analysis showed that: in terms of complications (OR: $0.65,95 \%$ CI: 0.13 to 3.00 , probability rank $=0.37$ ) and mitral regurgitation (OR:0.03, 95\% CI: 0.0 to 8315 , probability rank $=0.64$ ), the $3 \mathrm{D}-\mathrm{MVS}$ group had the lowest event rate. In terms of blood transfusion rate (OR: $0.55,95 \%$ CI: 0.16 to 1.84 , probability rank=0.45), T-MVS had the lowest event rate. In addition, with the exception of operation time and chest drainage, the R-MVS group has the best curative effect. Conclusion These minimally invasive surgery has their own advantages and disadvantages. Overall, 3DMVS is most satisfactory, but more samples are needed.

INPLASY registration number 202140138

\section{INTRODUCTION}

Mitral regurgitation (MR) is a common heart valve disease, in which the mitral valve fails to close during the left ventricular systole and causes blood to flow from the left ventricle to the left atrium, including primary (degenerative) MR and secondary (functional) MR. ${ }^{[1]}$ With the gradual aging of the global population, the number of MR patients has shown an upward trend year by year and the annual mortality rate was as high as $34 \% .{ }^{[2]}$ The treatment methods of MR mainly include drug therapy, surgery and percutaneous interventional therapy. Drug therapy can only improve the symptoms of the patient, but cannot prolong the survival time; the surgical operation includes mainly valve repair and valve replacement, which is considered to be the standard treatment for MR. Percutaneous intervention technology has become a supplementary treatment option for high-risk MR patients who cannot withstand the blow of surgery. ${ }^{[3]}$ After 30 years of development, Minimally invasive cardiac surgery (MICS) technology reduces the trauma caused by the operation itself in the case of providing the same curative effect as the C-MVS operation. In recent years, there have been studies that directly compared different minimally invasive options, such as R-MVS, 3D-MVS, T-MVS, MMVS, C-MVS, etc., but lack of network meta-analysis research. This study has been used to systematically evaluates the respective advantages and mid-term efficacy of different surgical procedures, discuss the best minimally invasive MV surgery and provide clinical reference for the majority of cardiac surgery colleagues.

\section{MATERIALS AND METHODS}

Patient and public involvement No patients were involved.

\section{Eligibility criteria}

Studies that meet the following inclusion criteria were included: (1) the design of the study was a clinical retrospective cohort study about MR; (2) The experimental group and the control group received different surgical treatments; and (3) at least four of the data indicators for the perioperative period. Exclusion criteria were as follows: (1) irrelevant studies and duplicate literature; (2) unavailable data in the literature;(3) letters, reviews, case reports. (4) The type of disease involves other major diseases that may affect the efficacy of the operation.

\section{Search methodology}


PubMed, EMBASE, The Cochrane Library, CNKI and VIP were comprehensively searched up to December 2020. The search terms were a combination of medical subject headings (MeSH) terms and the following free words: Mitral Regurgitation, Mitral Valve Replacement, Mitral Valve Repair, Endoscopes, Thoracoscopes, Robot-assisted, Robotic Surgical Procedures, Minimally Invasive Surgical Procedures, Thoracotomy. In addition, we manually searched for other potential and relevant references. There were no limitations in the language of all publications. Take PubMed as an example, its strategy is as follows:

$$
\text { \#1 Mitral regurgitation [Mesh] }
$$

\#2 Mitral valve replacement [Title/Abstract] OR Mitral valve repair [Title/Abstract] OR Mitral valvuloplasty [Title/Abstract]

\#3 \#1 and \#2

\#4 surgical therapy [Mesh]

\#5 Robotic Surgical Procedures [Mesh] OR Video-Assisted Surgery [Mesh] OR Thoracoscopes [Mesh] OR Thoracotomy [Mesh]

\#6 totally thoracoscopic [Title/Abstract] OR Traditional thoracotomy [Title/Abstract] OR Conventional Surgery [Title/Abstract]

\#7 \#4 OR \#5 OR \#6

\#8 \#3 AND \#7

\section{Study selection}

Two investigators (WMH and $\mathrm{BH}$ ) filtered the original studies independently. If the literature meets the eligibility criteria, the two investigators will further read the full text to screen the study. Any discrepancies were addressed by discussion or third party consensus.

\section{Data extraction and analysis}

All data were collected independently by two investigators (WMH and QL) from eligible studies using a standardised form. The following information was extracted: (1) study identification, including author name and publication year; (2) country where the study was conducted; (3) study subjects, number of participants and male to female ratio; (4) settings of trial arms; (5) Main indicators: complications, blood transfusion rate, 30-day mortality; mitral regurgitation and paravalvular leakage; secondary indicators: operation time, mechanical ventilation time, chest drainage, physiological score, psychological score, recovery time, hospitalization Total cost. (The "complication" here refers to postoperative wound infection, pneumonia, liver and kidney insufficiency, myocardial infarction, postoperative atrial fibrillation, stroke, secondary thoracotomy, etc., "Physiological score or Psychological score" is a score determined according to the quality of life questionnaire (SF-12/SF-36) provided in the literature. The higher the score, the better the effect.)

\section{Quality assessment}

Risk of bias was assessed using the Cochrane Collaboration's tool addressing six domains: sequence generation, allocation concealment, blinding of participants/outcome assessors, incomplete outcome, selective outcome reporting and other source of bias. These articles are almost non-randomized controlled (NRCT) studies, so the Newcastle Ottawa scale (NOS) recommended by Cochrane Collaboration Network is used to evaluate the quality of retrospective cohort studies.

\section{Statistical analysis}

Traditional pairwise meta-analysis with Review Manager 5.4.1For binary data, odds ratio (OR) and 95\% CI are used as effect size indicators. OR $<1$ indicates that one intervention may be worse than another intervention; OR $>1$ indicates that one intervention may be better than another intervention; $95 \% \mathrm{CI}$ containing 1 means that the difference is not statistically significant. For continuous data, standard mean difference 
(SMD) and $95 \%$ CI were used as effect size indicators. SMD $<0$ means that one intervention may be worse than another; SMD $>0$ means that one intervention may be better than another; $95 \%$ CI containing 0 means that the difference is not statistically significant. In direct Meta analysis, Q test and I2 index are used to evaluate the heterogeneity of each effect size. If $\mathrm{P}>0.1$ and $\mathrm{I} 2<50 \%$, it indicates that the results of each study have good homogeneity, then the fixed effects model is used. If P[?]0.1 and/or I2[?]50\%, the results of the study are statistically heterogeneous, and a random effects model is used.

Network meta-analysis with software ADDIS 1.16.8

The Node-split model is used to test the consistency in the network meta-analysis. If there is no statistical difference between the studies within the subgroup $(\mathrm{P}>0.05)$, it indicates that the heterogeneity of the included studies is small, so the consistency model is used for analysis; otherwise, an inconsistency model is used for analysis. A ranking probability table is used to rank the pros and cons of intervention measures (the value indicates the probability of intervention measures in the nth position). Regarding the main indicators of this article, the higher the ranking, the better.

\section{RESULTS}

\section{Study selection outcome and characteristics}

There were 1892 relevant articles retrieved ultimately, among which 272 were duplicate articles. After screening layer by layer, 31 ${ }^{[4-33]}$ articles were finally included, including 24 in English and 7 in Chinese, including 18 propensity matching studies and 4 master's thesis. Figure 1 shows the screening process.

The mean age of the included 12998 participants was 51.60 years. The follow-up time range is 1-50.5 months. Table 1 displays the main characteristics in detail.

\section{Study quality assessment}

The quality scores of the included observational studies were ranged from 5 to 8 with a mean score of 6.5 , which suggested the relatively high quality in the meta analysis (Table 2, in online supplement 1). All studies did not mention the allocation concealment and most of studies did not Provide the information about the blinding method (in clinical surgery, family members are required to obtain informed consent and inform their plans). The lowest agreement was achieved in the incomplete outcome data, while the perfect agreement was achieved in the selective reporting. The risk of bias of each study was showed in Figures 2 A,B.

Primary outcomes

In terms of complications, the $\mathrm{P}$ values of Node-splitting analysis are all $>0.05$, which means that the difference is not statistically significant. Therefore, the consistency test is used, and the probabilistic ranking results (Figure $4 \mathrm{~A}$ ) are in order: 3D-MVS $>$ M -MVS $>$ T-MVS $>$ R-MVS $>$ C-MVS. Specific values: 3DMVS(OR:0.65, 95\%CI:0.13 to 3.00), M-MVS(OR=0.56, 95\%CI:0.35 to 0.90) )], T-MVS (OR: 0.60, $95 \%$ CI:0.32 to 1.15), R-MVS (OR: 0.76, 95\% CI:0.48 to 1.14)) are less than C-MVS.

In terms of blood transfusion rate, the ranking result is: T-MVS $>3$ D-MVS $>$ M-MVS $>$ R-MVS $>$ C-MVS. Specific value: T-MVS(OR:0.31, 95\%CI:0.07 to 1.40), 3D -MVS(OR:0.35, 95\%CI:0.05 to 2.62), M-MVS(OR:0.61, 95\% CI:0.27 to 1.34), R-MVS(OR:0.77, 95\%CI:0.38 to 1.55$)$ Lower than C-MVS.

In terms of MR and paravalvular leakage, the ranking result is: 3D-MVS $>$ M-MVS $>$ T-MVS $>$ R-MVS $>$ CMVS. The specific value is: 3D-MVS(OR:0.03, 95\%CI:0.00 to 8315 ), M-MVS(OR: 0.46, 95\%CI: 0.25 to 0.88), T-MVS(OR: $0.64,95 \% \mathrm{CI}: 0.25$ to 1.47 ), R-MVS(OR: 0.69, 95\%CI: 0.35 to 1.24 ) are lower than C-MVS.

Regarding the 30-day mortality rate, we use the node-split model (NM) for non-uniformity test. The last line indicates $\mathrm{P}<0.05$, that is, the difference is statistically significant. According to the principles of statistical methods, we use non-uniformity test. Consistency test (Figure 5) and analysis of the source of inconsistency. The inconsistency suggests that there is a significant statistical difference between direct comparison and indirect comparison, so we should refer to the traditional direct comparison result to be more reliable. 


\section{Secondary outcomes}

In terms of mechanical ventilation time, the robot takes the shortest time, and the ranking result is: $\mathrm{R}$ MVS $>3$ D-MVS $>$ T-MVS $>$ M-MVS $>$ C-MVS.

In terms of recovery time, the robot group recovers the fastest, and the ranking results are: R-MVS $>$ MMVS $>$ T-MVS $>$ C-MVS.

In terms of postoperative thoracic drainage, the small incision drainage value is the smallest, and the ranking result (from less to more drainage) is: M-MVS $>$ T-MVS $>3 D-M V S>R-M V S>C-M V S$.

In terms of scoring, only 4 intervention measures were compared. In terms of physiological score, the ranking results of superiority and inferiority were as follows: R-MVS $>$ M-MVS $>$ T-MVS $>$ C-MVS. In terms of psychological score, the order is: R-MVS > T-MVS >C-MVS>M-MVS.

\section{Publication bias}

A funnel plot of all included studies in this network meta-analysis was made for visual screening of any publication bias (figure 6). It revealed that all included studies were distributed around the vertical and oblique line within the $95 \%$ CIs, suggesting no obvious publication bias.

\section{DISCUSSION}

The above-mentioned surgical procedures have their own advantages and disadvantages, and even C-MVS is still used as a routine operation method in some institutions restricted by endoscopic conditions due to its remarkable curative effect. ${ }^{[27]}$ The effect of different surgical methods in the treatment of mitral regurgitation is described, and the probabilistic results of their pros and cons are given.

It should be emphasized that in terms of total hospitalization costs, three of the studies ${ }^{[7,23,27]}$ involved and performed statistical analysis, among which Zhu Yilin ${ }^{[27]}$ ( $\mathrm{P}=0.391$, T-MVS vs M-MVS vs C- MVS), Coyan $^{[7]}(\mathrm{P}=0.273$, R-MVS vs C-MVS) directly compared the results with no statistical difference; while Liu's study ${ }^{[23]}$ suggested that the difference was statistically significant ( $\mathrm{P}=0.002$, $\mathrm{T}$-MVS vs C-MVS). Perin $^{[34]}$ and others abroad reported that the total hospitalization cost of minimally invasive and C-MVS mitral valve surgery is not different: the high surgical cost of minimally invasive surgery is lowered by the lower postoperative cost. The cost is offset, and the length of hospital stay is shortened by about 2 days, which is consistent with the author's research results to a certain extent. In addition, we can conclude that the minimally invasive surgery options can reduce trauma to a certain extent by comparing them with C-MVS. Saving blood products, reducing complications, and speeding up body recovery are beneficial to improve the quality of life of patients after surgery.

Although most of articles record the left ventricular ejection fraction (LVEF) values before, due to the large clinical heterogeneity between the studies, the postoperative EF value was not included in the outcome indicators for comparison. A Meta study in China ${ }^{[35]}$ once reported that the Percutaneous Mitral Valve Clipping (Mitra-Clip treatment) can improve the cardiac function of patients with mitral regurgitation and heart failure to a certain extent, but further studies are still needed to prove its conclusions; recent studies by Khader ${ }^{[36]}$ and others in the UK have also repeatedly emphasized the mid-term durability of MR valve surgical repair is better than Mitra-Clip.

Combined with this Network Meta, we believes that MR minimally invasive surgery will still be the standard treatment plan for mitral valve disease for a long period of time in the future. Which minimally invasive program needs to be determined by the institution based on the individual and the environment.

\section{CONCLUSION}

Based on the above network meta-analysis and ranking results, 3D-MVS operation method can be used as the best choice in the surgical scheme of mitral valve disease, followed by T-MVS and M-MVS. Of course, if there are resource conditions, the combination of two minimally invasive operations (such as small incision combined with thoracoscopy) can ensure safer operation and faster recovery of patients. 
Figure 1 Study flow diagram

Figure 2 A-B. (A) risk of bias summary; (B) risk of bias graph.

Figure 3 a-d. Network diagram of the treatments. (a ): Complications; (b ): Blood transfusion rate; (c) : Mitral regurgitation and perivalvular leakage during follow-up; (d ): Mortality within 30 days.

Figure 4 A-C. Rank probability of the best treatment. Rank 1 is worst, rank $\mathrm{N}$ is best. (A): Complications; (B): Blood transfusion rate; (C): Mitral regurgitation and perivalvular leakage during follow-up.

Figure 5 Node-splitting analysis of the 30-day mortality index. Data are presented as relative effect (95\% credible interval).

Figure 6 A funnel plot of all included studies in this network meta-analysis was made for visual screening of any publication bias.

\section{Author affiliations}

1. Baotou Clinical Medical College of Inner Mongolia Medical University, Baotou, China

2. Department of Cardiology, Baotou Central Hospital, Baotou 014040, China

Acknowledgements Our greatest acknowledgement goes to the authors who made detailed data available for this meta-analysis and all our colleagues in this study for their hard work.

Contributors WMH, BH and QL performed the search and drafted the manuscript. WMH and YHZ performed the data extraction and analysed the data. WMH, WL and YHZ designed the study and amended the original draft.

Funding The authors have not declared a specific grant for this research from any funding agency in the public, commercial or not-for-profit sectors.

Competing interests None declared.

Patient and public involvement Patients and/or the public were not involved in the design, or conduct, or reporting, or dissemination plans of this research.

Patient consent for publication Not required.

Provenance and peer review Not commissioned; externally peer reviewed.

Data availability statement All data relevant to the study are included in the article or uploaded as supplementary information.

ORCID iD Weimin Huanghttps://orcid.org/0000-0003-2556-6214

\section{REFERENCES}

[1] Walther C, Fichtlscherer S, Holubec T, et al. New developments in transcatheter therapy of mitral valve disease[J]. J Thorac Dis , 2020,12(4):1728-1739.

[2] Welp H, Martens S. Minimally invasive mitral valve repair[J]. Curr Opin Anaesthesiol , 2014,27(1):65-71.

[3] Fei F, Zhao H, Qu L L, et al. Zeng Zhi: analysis of 2017 global expert consensus on percutaneous interventional treatment of mitral regurgitation [J]. Chinese medical science, 2017,7 (11): 1-2

[4] Paparella D, Fattouch K, Moscarelli M, et al. Current trends in mitral valve surgery: A multicenter national comparison between full-sternotomy and minimally-invasive approach[J]. Int J Cardiol , 2020,306:147151.

[5] Seo Y J, Sanaiha Y, Bailey K L, et al. Outcomes and Resource Utilization in Robotic Mitral Valve Repair: Beyond the Learning Curve[J]. J Surg Res , 2019,235:258-263. 
[6] Hawkins R B, Mehaffey J H, Mullen M G, et al. A propensity matched analysis of robotic, minimally invasive, and conventional mitral valve surgery[J]. Heart , 2018,104(23):1970-1975.

[7] Coyan G, Wei L M, Althouse A, et al. Robotic mitral valve operations by experienced surgeons are cost-neutral and durable at 1 year[J].J Thorac Cardiovasc Surg , 2018,156(3):1040-1047.

[8] Kesävuori R, Raivio P, Jokinen J J, et al. Early experience with robotic mitral valve repair with intraaortic occlusion[J]. The Journal of thoracic and cardiovascular surgery, 2018,155(4):1463-1471.

[9] Wang A, Brennan J M, Zhang S, et al. Robotic Mitral Valve Repair in Older Individuals: An Analysis of The Society of Thoracic Surgeons Database[J]. Ann Thorac Surg , 2018,106(5):1388-1393.

[10] Lange R, Voss B, Kehl V, et al. Right Minithoracotomy Versus Full Sternotomy for Mitral Valve Repair: A Propensity Matched Comparison[J].Ann Thorac Surg , 2017,103(2):573-579.

[11] De Bonis M, Lapenna E, Del F B, et al. Minimally invasive or conventional edge-to-edge repair for severe mitral regurgitation due to bileaflet prolapse in Barlow's disease: does the surgical approach have an impact on the long-term results?[J]. Eur J Cardiothorac Surg, 2017,52(1):131-136.

[12] Nishi H, Miyata H, Motomura N, et al. Propensity-matched analysis of minimally invasive mitral valve repair using a nationwide surgical database[J]. Surg Today , 2015,45(9):1144-1152.

[13] Westhofen S, Conradi L, Deuse T, et al. A matched pairs analysis of non-rib-spreading, fully endoscopic, mini-incision technique versus conventional mini-thoracotomy for mitral valve repair $[\mathrm{J}]$. Eur $J$ Cardiothorac Surg , 2016,50(6):1181-1187.

[14] Goldstone A B, Atluri P, Szeto W Y, et al. Minimally invasive approach provides at least equivalent results for surgical correction of mitral regurgitation: a propensity-matched comparison[J]. J Thorac Cardiovasc Surg , 2013,145(3):748-756.

[15] Stevens L M, Rodriguez E, Lehr E J, et al. Impact of timing and surgical approach on outcomes after mitral valve regurgitation operations[J].Ann Thorac Surg , 2012,93(5):1462-1468.

[16] Suri R M, Burkhart H M, Daly R C, et al. Robotic mitral valve repair for all prolapse subsets using techniques identical to open valvuloplasty: establishing the benchmark against which percutaneous interventions should be judged[J]. J Thorac Cardiovasc Surg , 2011,142(5):970-979.

[17] Mihaljevic T, Jarrett C M, Gillinov A M, et al. Robotic repair of posterior mitral valve prolapse versus conventional approaches: potential realized[J]. J Thorac Cardiovasc Surg, 2011,141(1):72-80.

[18] Speziale G, Nasso G, Esposito G, et al. Results of mitral valve repair for Barlow disease (bileaflet prolapse) via right minithoracotomy versus conventional median sternotomy: a randomized trial[J]. J Thorac Cardiovasc Surg , 2011,142(1):77-83.

[19] Huang H L, Yan Q, Xie X J, et al. Early outcomes of mitral valvuloplasty by minimally invasive surgery or sternotomy[J]. Asian Cardiovasc Thorac Ann, 2020,28(7):398-403.

[20] Jiang Q, Wang Z, Guo J, et al. Retrospective Comparison of Endoscopic Versus Open Procedure for Mitral Valve Disease[J]. J Invest Surg, 2020:1-7.

[21] Zang X, Huang H L, Xie B, et al. A comparative study of three-dimensional high-definition and twodimensional high-definition video systems in totally endoscopic mitral valve replacement[J]. J Thorac Dis , 2019,11(3):788-794.

[22] Fan H, Xi M Q, Wei M Z, et al. Comparison of Totally Thoracoscopic and Traditional Sternotomy Approaches for Mitral Valve Replacement[J].Heart Surg Forum, 2019,22(4):E310-E314.

[23] Liu J, Chen B, Zhang Y Y, et al. Mitral valve replacement via minimally invasive totally thoracoscopic surgery versus traditional median sternotomy: a propensity score matched comparative study[J].Ann Transl Med, 2019,7(14):341. 
[24] Wang Q, Xi W, Gao Y, et al. Short-term outcomes of minimally invasive mitral valve repair: a propensitymatched comparison[J]. Interact Cardiovasc Thorac Surg , 2018,26(5):805-812.

[25] Zhao G, Gao J, Liu Y, et al. Two-Incision Totally Thoracoscopic Approach for Mitral Valve Replacement[J]. Int Heart $J$, 2017,58(6):894-899.

[26] Wei L, Shen J Q, Wang C S, et al. [Repair of posterior mitral valve prolapsed: comparative study of three different approaches][J].Zhonghua Wai Ke Za Zhi, 2012,50(7):637-641.

[27] Zhu Y L. Comparison of mitral valvuloplasty with total thoracoscopy and right anterolateral small incision [D].Lanzhou University, 2020

[28] Liu Y, Zhang H, Hu H Y, et al. Comparative study on the near and medium-term efficacy of Da Vinci robot assisted and traditional mitral valvuloplasty $[\mathrm{J}]$. Chinese Journal of thoracic and cardiovascular surgery , 2020,27 (10): 1161-1167

[29] Zhao H Z, Zhang H J, Yang M, et al. Comparison of quality of life and long-term efficacy between robot assisted and median sternotomy mitral valve replacement [J]. Journal of Southern Medical University , 2020,40 (11): 1557-1563

[30] Yin L M. Clinical comparative study of Da Vinci robot assisted and conventional thoracotomy mitral valvuloplasty [D]. Qingdao University, 2019

[31] Han L. Comparative study of mitral valve replacement by total thoracoscopy and right thoracic small incision [D]. Lanzhou University, 2018

[32] Fu Z K, Zhang X L, Wang L C, et al. Application of 3D thoracoscopy in mitral valve surgery [J]. Chinese Journal of cardiopulmonary bypass , 2018,16 (01): 21-24

[33] Yuan N N. Comparative study on medium-term shaping effect and quality of life between full robot mitral valvuloplasty and traditional thoracotomy [D]. Medical College of the Chinese people's Liberation Army, 2013

[34] Perin G, Shaw M, Toolan C, et al. Cost Analysis of Minimally Invasive Mitral Valve Surgery in the UK National Health Service[J]. The Annals of Thoracic Surgery , 2021,112(1).

[35] Jiang Z Z, Liu Z, Cheng X C. Meta analysis of the efficacy of percutaneous mitral valve clipping in patients with mitral insufficiency complicated with heart failure $[\mathrm{J}]$. Chinese Journal of evidence based medicine, 2016,16 (04): 409-414

[36] Khader A A, Allaf M, Lu O W, et al. Does the clinical effectiveness of Mitraclip compare with surgical repair for mitral regurgitation?[J].J Card Surg , 2021,36(3):1103-1119. 


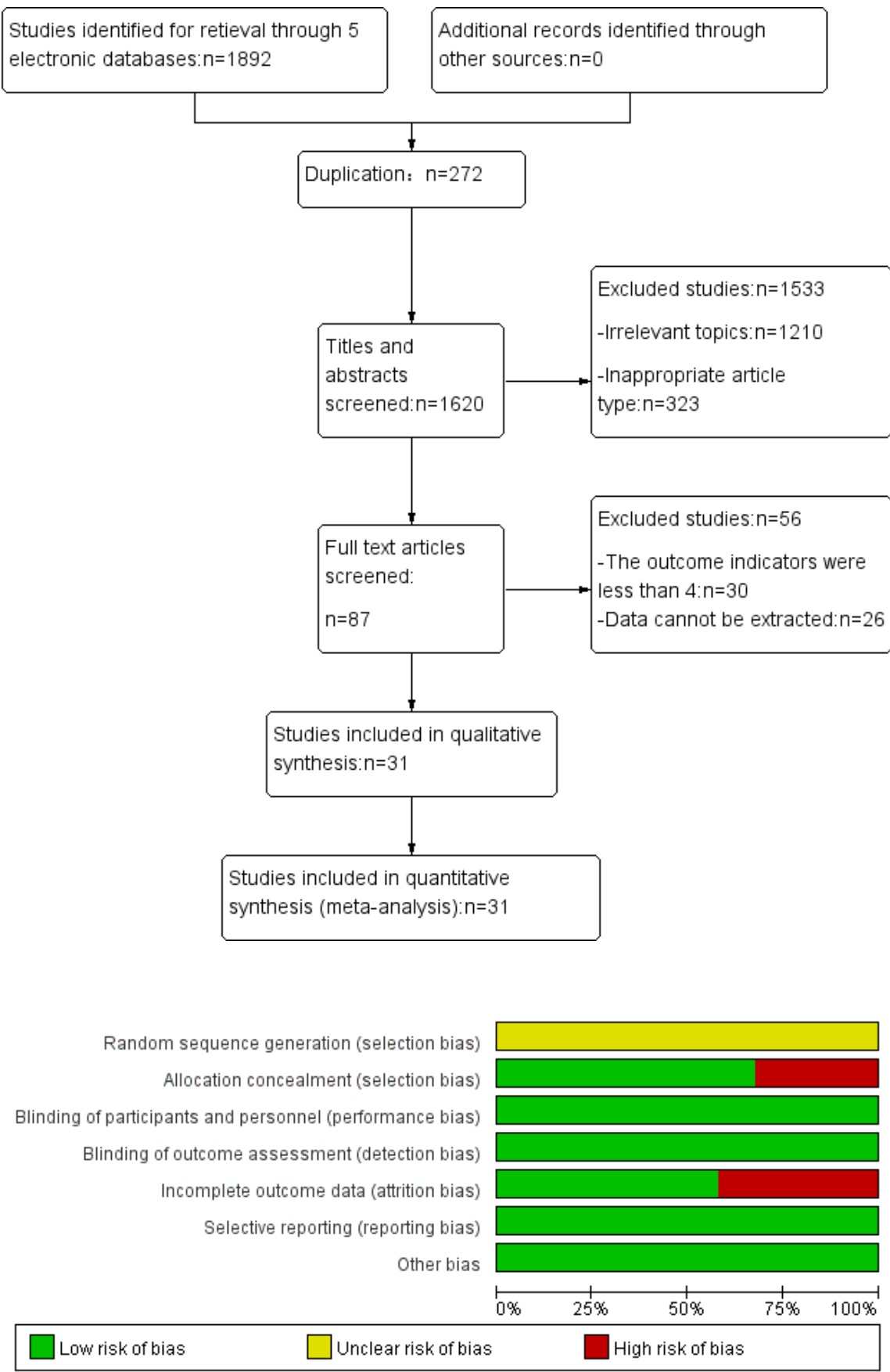




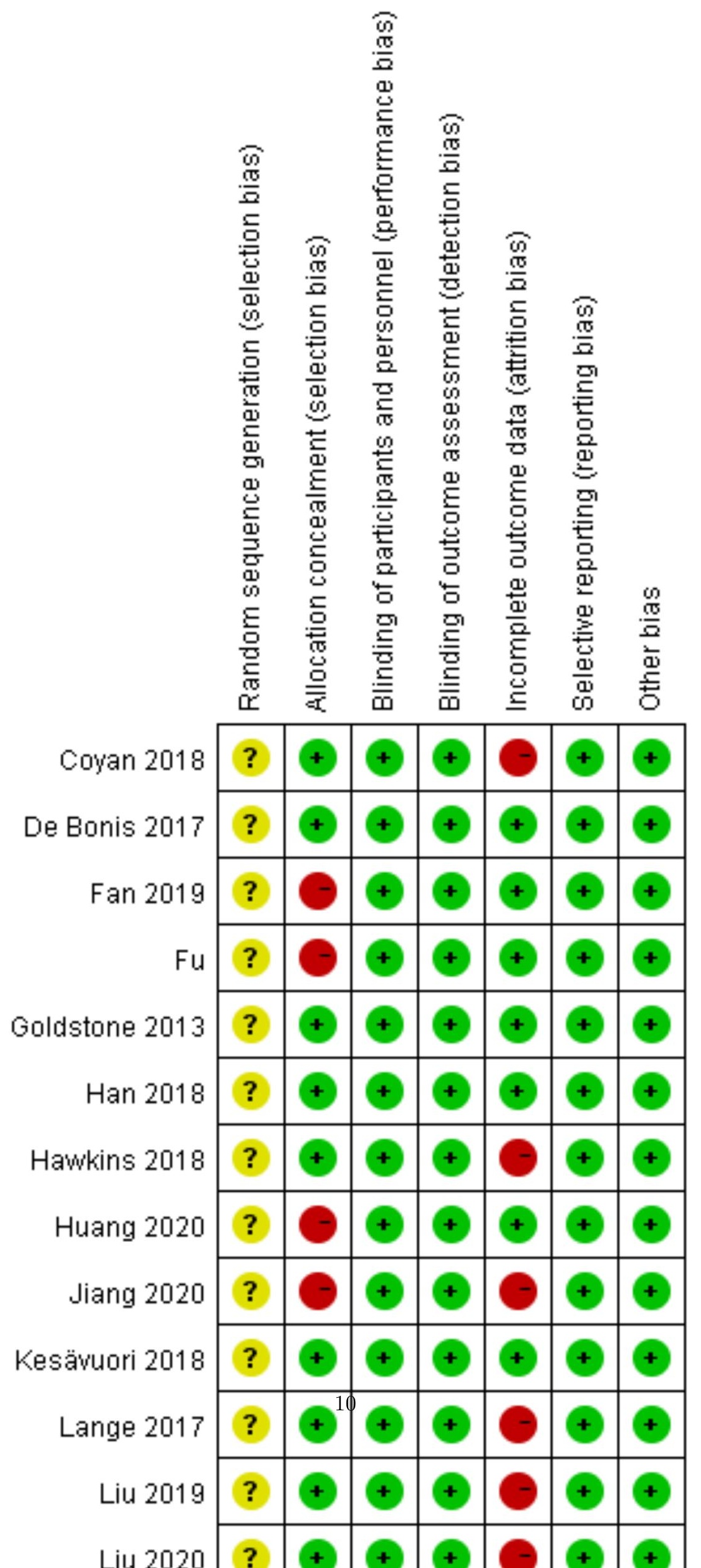



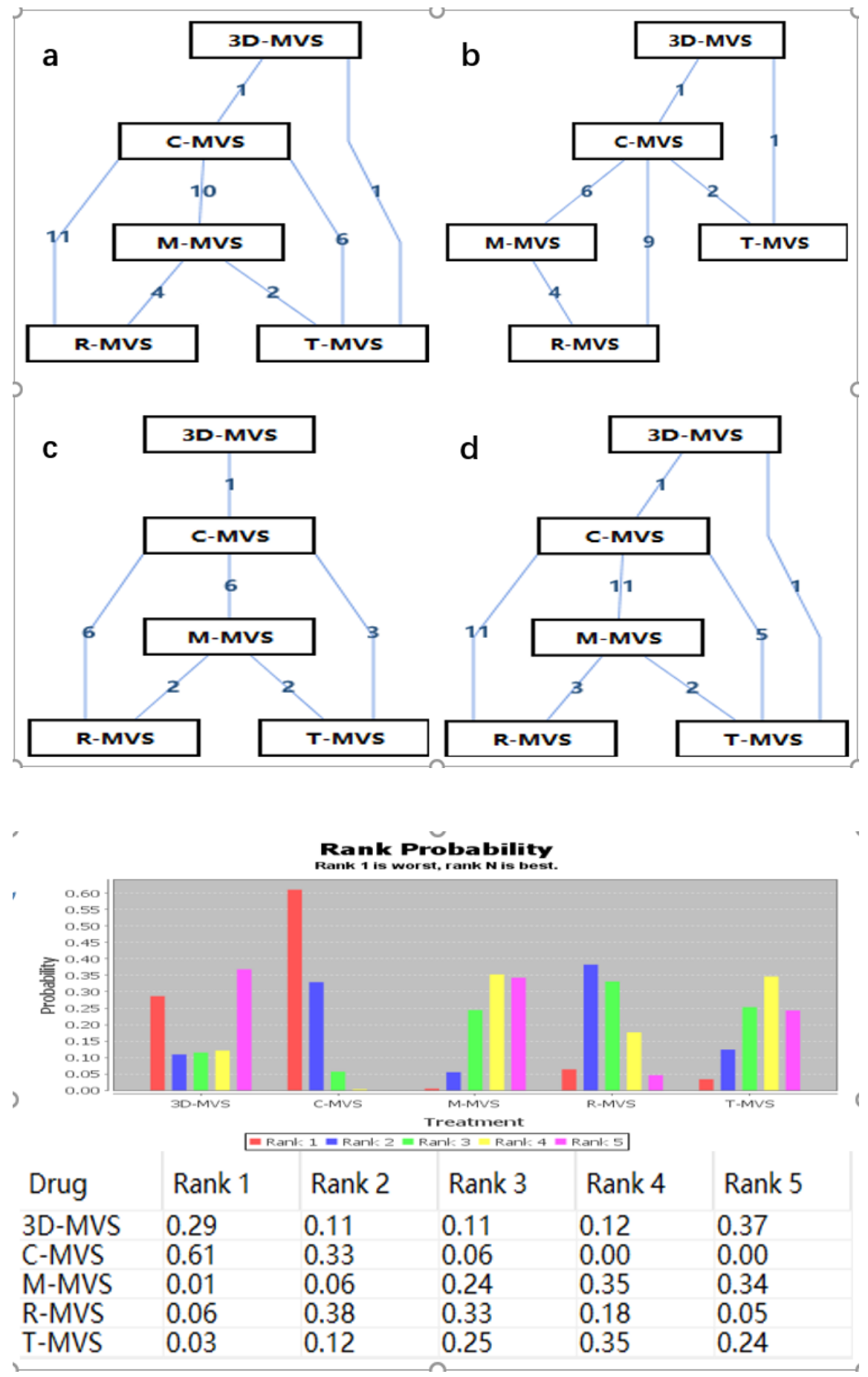

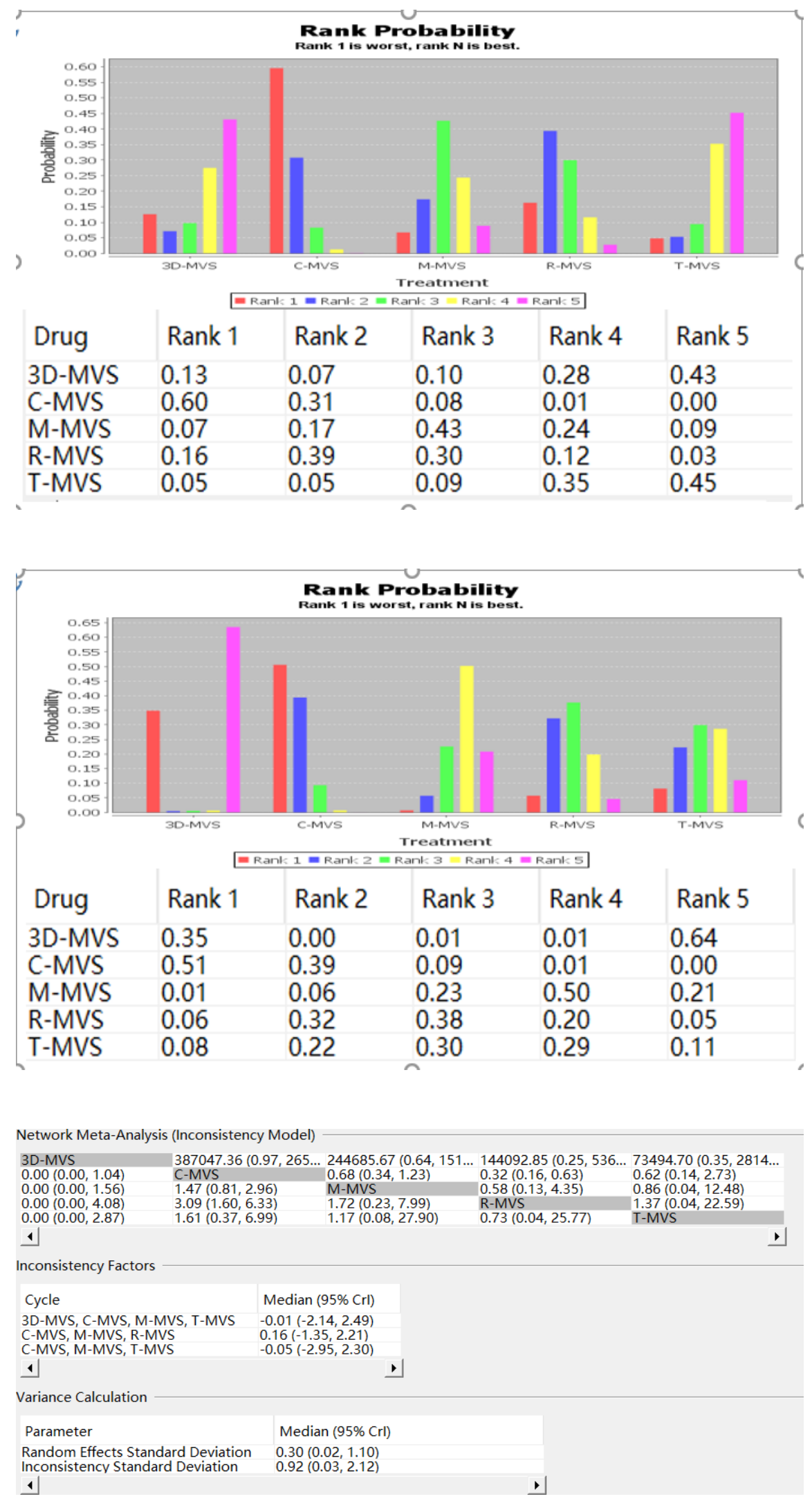


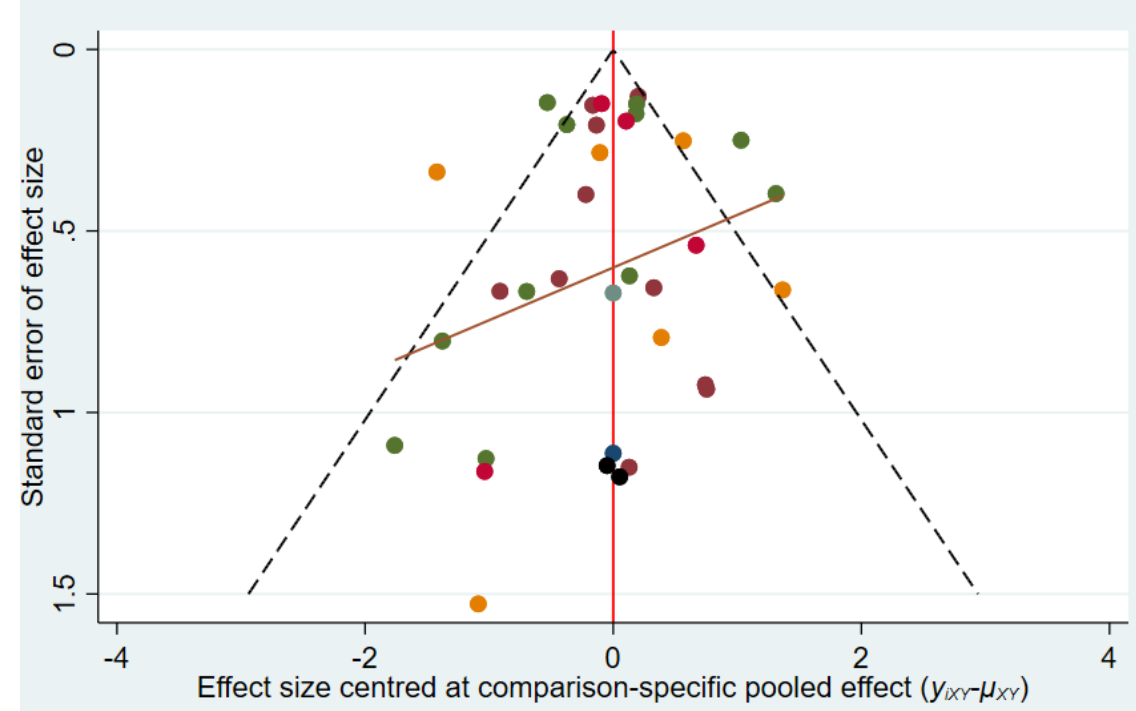

- Avs B A Avs C A Avs D AvsE B B E $\bullet$ C vs D

- C vs E

\section{Hosted file}

Table 1 Main information of the studies included in the meta.docx available at https: //authorea.com/users/435929/articles/538623-comparative-efficacy-of-5-surgical-methodsin-the-treatment-of-mitral-regurgitation-a-systematic-review-and-network-meta-analysis 